\title{
Studying Rigorously Defined Health Care Processes Using a Formal Process Modeling Language, Clinical Simulation, Observation, and Eye Tracking
}

\author{
Jenna L. Marquard \\ College of Engineering \\ University of Massachusetts, Amherst \\ marquard@engin.umass.edu \\ Philip L. Henneman \\ Department of Emergency Medicine \\ Baystate Medical Center \\ Tufts University School of Medicine \\ philip.henneman@bhs.org
}

\section{Leon J. Osterweil}

Department of Computer Science

University of Massachusetts, Amherst ljo@cs.umass.edu

\author{
Donald L. Fisher \\ College of Engineering \\ University of Massachusetts, Amherst \\ fisher@ecs.umass.edu
}
Megan M. Campbell
School of Nursing
University of Massachusetts, Amherst mcampbel@student.umass.edu

Qi Ming Lin

College of Engineering

University of Massachusetts, Amherst

qlin@student.umass.edu

\author{
Stefan Christov \\ Department of Computer Science \\ University of Massachusetts, Amherst \\ christov@cs.umass.edu \\ Lori A. Clarke \\ Department of Computer Science \\ University of Massachusetts, Amherst \\ clarke@cs.umass.edu
}

George S. Avrunin

Department of Mathematics and Statistics

University of Massachusetts, Amherst

avrunin@math.umass.edu

\section{Elizabeth A. Henneman}

School of Nursing

University of Massachusetts, Amherst

henneman@nursing.umass.edu

\section{Tuan A. Pham}

College of Engineering

University of Massachusetts, Amherst

tpham@student.umass.edu

ABSTRACT

Motivation - The complex nature of health care processes requires new methods for describing, capturing and improving these processes. Research approach - We deployed a novel combination of methods - formal process modeling using a language called Little-JIL, simulations with embedded errors, observations, and eye tracking technology - to gauge how health care providers complete one complex process, patient identification. Findings/Design - These methods allowed us to thoroughly analyze how health care providers completed the patient identification process with and without embedded errors, and to record exactly what participants looked at during the simulations. Research limitations/Implications - We have used this set of methods to analyze only one type of health care process to-date. Originality/Value - We can use these approaches to inform health care provider training, process redesign, and the design of technologies to support health care providers as they verify patients' identities.

\section{Keywords}

Process modeling, clinical simulation, eye tracking, patient identification

\section{INTRODUCTION}

Over the past several years, an interdisciplinary research team composed of computer scientists, industrial engineers (University of Massachusetts, Amherst) and health care researchers (Baystate Medical Center, Springfield, MA) has worked to address three general research questions:

1. Can the formal modeling language Little-JIL be used to model complex health care processes and support their analysis? Little-JIL allows modelers to define a process at any desired level of detail, including the specification of how exceptional, non-normative conditions are to be handled. This work has yielded rigorous and 
precise models of a chemotherapy process, a blood transfusion process, and providers' verification of a patient's identity (Chen, Avrunin, Henneman, Clarke, Osterweil, \& Henneman, 2008). Our experience indicates that these process models can serve as a basis for analyses (including several types of automated analyses) aimed at detecting and correcting modes of failure, thereby assisting decision making aimed at improving the process itself.

2. Can a novel combination of methods - eye tracking, observation and clinical simulation - be used to capture the details of what real individuals do as they complete complex health care processes? Eye tracking devices used to measure eye position and eye movement - have been widely used to evaluate individual performance by gaining insight into perception, attention, and other cognitive processes. Eye tracking devices prevent observer bias, allow for fairly unobtrusive evaluation of individual performance, and have been used successfully in research domains from transportation to medical practice (Fisher, Pradhan, Pollatsek, \& Knodler, 2007; Henneman et al., 2008). Additionally, participants with eye tracking devices are often placed in simulated settings where they encounter faults in automation, risky and/or complex scenarios, or embedded errors.

3. Can algorithms be developed and used to compare how real individuals actually perform a complex process to a model of their performance as expressed in Little-JIL? A Little-JIL process model is often created by computer scientists who elicit the process from a group of domain experts. The model might represent an "ideal" process or a process biased by the experience of that particular group - and thus might not be an accurate reflection of how the process is actually performed by health care providers. To improve the usefulness of formal process modeling, we must develop and use algorithms to compare how real individuals perform a complex process (from eye-tracking and observational data, for instance) to a process model created in Little-JIL.

\section{METHOD}

In this poster, we focus on how health care providers perform the process of verifying a patient's identity (ID). To understand how health care providers actually perform this process, we engaged them in realistic but simulated clinical scenarios. Three types of Emergency Department health care providers completed the simulated scenarios and each was asked to perform a task familiar to them: emergency service associates (ESAs) placed an ID band on a patient, technical associates (TAs) drew and labeled a patient's blood specimen, and registered nurses (RNs) administered an intravenous medication to a patient. All of these tasks required the care provider to verify the patient's ID prior to performing the task. Each care provider completed three simulations, but in all cases, the third simulation entailed having to recognize and deal with an ID error that was intentionally embedded in the simulation. While participating in the simulations, the health care providers wore an eye tracker device and were observed by an individual who recorded their specific actions. We evaluated the use of these techniques as vehicles for validating the degree to which health care provider actions were consistent with the process modeled in Little-JIL - which was created via input from a domain expert.

\section{RESULTS}

The use of the research methods described above provided several insights into how different health care providers actually perform the process of patient identification. First, the combination of observation and eye-tracker technology allowed us to map specific physical manifestations of the process steps (e.g. looking at specific ID information) to the cognitive process (e.g. verification) outlined in the Little-JIL process model. Second, by having both simulations with and without embedded errors we could assess how health care providers performed a typical version of the process (i.e. patients $1 \& 2$ without an ID error) and how they performed the process under exceptional conditions (i.e. patient 3 with embedded ID error). We could also assess whether health care providers who identified the ID error in patient 3 performed the process differently from participants who did not catch the ID error.

\section{DISCUSSION}

The formal process modeling language, Little-JIL, can rigorously and precisely model complex health care processes. Specific methods usefully gauge how health care providers actually complete the process of patient identification; namely simulations with embedded errors, observations, and eye-tracker technology to record exactly what the participants were looking at during the simulations. These approaches can inform health care provider training, process re-design, and the design of technologies to support health care providers as they verify patients' identities.

\section{REFERENCES}

Chen, B., Avrunin, G. S., Henneman, E. A., Clarke, L. A., Osterweil, L. J., \& Henneman, P. L. (2008). Analyzing medical processes. ICSE '08: Proceedings of the 30th International Conference on Software Engineering, 623-32.

Fisher, D. L., Pradhan, A. K., Pollatsek, A., \& Knodler, M. A. (2007). Empirical Evaluation of Hazard Anticipation Behaviors in the Field and on Driving Simulator Using Eye Tracker. Transportation Research Record, 2018(1), 8086.

Henneman, P. L., Fisher, D. L., Henneman, E. A., Pham, T. A., Mei, Y. Y., Talati, R., Nathanson, B. H., \& Roche, J. (2008). Providers do not verify patient identification during computer order entry. Acad Emerg Med, 15(7), $641-648$. 